\title{
Estudio endoscópico en familiares de primer grado de pacientes operados por cáncer colorrectal
}

\author{
Francisco López-Köstner, Demian A Fullerton, \\ $U$ do Kronberg, G onzalo Soto $D$, Álvaro Zúñiga $D$, \\ Johanna Argandoña ${ }^{a}$, Vanesa Miranda Ba, Eliana Pinto Ta. \\ Screening colonoscopy among \\ first degree relatives of patients \\ with colorectal carcinoma
}

Background: First degree relatives of patients with colorectal carcinoma are at a higher risk of having the disease than the general population. Therefore, they should be subjected to screening colonoscopy. Aim: To assess the effectiven ess of colonoscopy among first degree relatives of patients with colorectal carcinoma. Material and methods: A free colonoscopy was offered to first degree relatives of patients operated on for colorectal cancer between 1998 and 2000. As inclusion criteria, subjects had to be asymptomatic, older than 40 years or less than 10 years younger than the index case. Each subject was contacted twice, inviting him/her to have a colonoscopy performed. Results: Two hundred forty three relatives were contacted for the study and in 76, a colonoscopy was performed. Among the latter, a neoplasm was found in 13 (17\%): One adenocarcinoma and 12 adenomas. Three of these lesions were located in the right colon. The main reason given by the 176 subjects that did not agree to have a colonoscopy was lack of interest. Conclusions: Screening colonoscopy is effective to detect adenoma and adenocarcinomaamong first degree relatives of patients with colorectal carcinoma, however only $31 \%$ of all potential relatives agreed to undergo a colonoscopy (Rev Méd Chile 2006; 134: 997-1001).

(Key words: Colonic neoplasms; Colonoscopy; Rectal neoplasms)

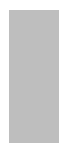

Recibido el 18 de marzo, 2005, Aceptado el 2 de marzo, 2006.

Departamento de Cirugía Digestiva, Facultad de Medicina, Pontificia Universidad Católica de Chile, Santiago, Chile.

aEnfermera universitaria

A 1 analizar la epidemiología del cáncer colorrectal (CC), se puede observar que los factores de riesgo no modificables (historia fami-

Correspondencia a: Dr. Francisco López Köstner. Departamento de Cirugía Digestiva, Facultad de Medicina, Pontificia Universidad Católica de Chile. Marcoleta 367, Santiago, Chile. Fax: 632 9620. E mail: flopez@med.puc.cl

liar, edad y antecedentes mórbidos asociados) son aquellos con evidencia más fuerte de causalidad. Los otros factores de riesgo que se mencionan (aquellos relacionados con la dieta, consumo de tabaco, alcohol, etc.) se sustentan en estudios epidemiológicos que, aunque muestran significación estadística, en conjunto demuestran resultados muchas veces contradictorios, con un control 
inadecuado de sesgos y revelando asociaciones que podrían ser indirectas ${ }^{1}$. Por esto, las políticas de prevención consideran principalmente estrategias orientadas al diagnóstico precoz, porque mientras no exista evidencia fuerte en cuanto al control de factores de riesgo modificables o el uso de quimioprevención, no se pueden plantear intervenciones en este terreno en la población general.

La incidencia del CC en países occidentales va en aumento, situación que también ocurriría en Chile $^{2}$. Sin embargo, Estados Unidos de Norteamérica (EE.UU.) ha demostrado una reducción en la incidencia y en la mortalidad de esta enferme$\mathrm{dad}^{3}$. Una explicación a este fenómeno podría estar en la incorporación de estrategias de detección precoz que permiten el diagnóstico y tratamiento de las lesiones premalignas ${ }^{1}$.

En ese país se han elaborado guías clínicas basadas en la revisión consensuada de la eviden$\mathrm{cia}^{4}$ que proponen que el screening del CC se inicie en la población general desde los 50 años. Aunque existe suficiente evidencia para afirmar que las estrategias de detección precoz son costoefectivas, aún no es posible afirmar cuál es la mejor modalidad de estudio a utilizar ${ }^{4-6}$. Los familiares de primer grado de pacientes con cáncer o adenomas colorrectales, constituyen un universo de mayor riesgo que el de la población general, en ellos existe una incidencia mayor de cáncer colorrectal y los tumores en el colon tienden a aparecer a más temprana edad. Por ello, se recomienda en este grupo iniciar la vigilancia de CC desde los 40 años, o desde los 10 años previos a la edad en que el sujeto índice se le diagnosticó el tumor ${ }^{4}$.

En nuestro país, la revisión de datos indirectos indica que cerca de 1.000 personas fallecen al año por cáncer colorrectal y que su prevalencia estaría aumentando ${ }^{2}$. El objetivo de este trabajo es definir la utilidad del estudio con colonoscopia en el grupo de alto riesgo representado por los familiares de primer grado de pacientes con cáncer colorrectal.

\section{MATERIAL Y MÉTODO}

Se diseñó un protocolo prospectivo, que comenzó con la identificación de los familiares de primer grado de los pacientes operados por CC entre abril de 1998 y abril de 2000. Para la inclusión de éstos en el estudio, se requirió que fueran mayores de 40 años o menores de 10 años a la edad en que su pariente presentó CC. Se exigió también que ninguno hubiera presentado síntomas de sospecha de esa enfermedad. Se constituyó un equipo de tres enfermeras que contactaron telefónicamente a los individuos seleccionados. Se efectuaron dos entrevistas telefónicas en las que se informó sobre la situación de riesgo, se constató la calidad de asintomático de los individuos y se les invitó a participar en el estudio a través de la realización de una colonoscopia sin costo. Las colonoscopias se efectuaron en un plazo de tres meses (mayo a julio de 2000).

La colonoscopia se efectuó después de una preparación con régimen líquido y administración de Fleet-Oral ${ }^{\circledR}$, en dos dosis, el día previo al examen. Se administró sedación intravenosa con midazolam y meperidina. El equipo examinador estuvo constituido por tres médicos endoscopistas del Laboratorio de Gastroenterología del Hospital Clínico de la Pontificia Universidad Católica de Chile.

El objetivo de la colonoscopia fue el examen del colon hasta el ciego. Ante el hallazgo de pólipos, éstos se extirparon de acuerdo a su tamaño con asa caliente o con pinza. Las muestras fueron analizadas en el Departamento de Anatomía Patológica del mismo hospital.

\section{Resultados}

El grupo de familiares contactado estuvo constituido por 243 individuos, ellos eran familiares vivos de primer grado de 165 pacientes operados por cáncer colorrectal entre 1998 y 2000. Cincuenta y cinco por ciento de los individuos eran mujeres.

En el momento de la entrevista telefónica, 48 personas asintomáticas ya se habían efectuado una colonoscopia en el Laboratorio de Gastroenterología, 107 se comprometieron a participar en el estudio y 88 personas (36\%) declinaron participar. Al interrogarles por el motivo por el cual no participarían, $71 \%$ dijo que no le interesaba o que no quería, y $20 \%$ rechazó por ser individuos de edad avanzada.

De los 107 que se comprometieron a asistir, sólo 28 acudieron al examen. Al grupo que 
Tabla 1

\begin{tabular}{|lrrrrr|}
\hline & \multicolumn{2}{c}{ Causas por las que no } & & \multicolumn{2}{c|}{ Causas de no adherencia en } \\
& se comprometen a participar & & \multicolumn{2}{c|}{ individuos que se habían comprometido } \\
& $\mathrm{n}$ & $\%$ & & $\mathrm{n}$ & $\%$ \\
\hline Miedo & 2 & 2,3 & Miedo & 11 & 13,9 \\
Edad & 17 & 19,5 & Edad & 1 & 1,3 \\
No quieren & 63 & 71,3 & No quieren & 57 & 72,2 \\
Costo* & 6 & 6,9 & Costo* & 10 & 12,6 \\
Total & 88 & 100 & Total & 79 & 100 \\
\hline
\end{tabular}

*Algunos pacientes se negaron a participar por razones de costo. El estudio incluyó el pago completo de la colonoscopia y de las biopsias. No cubrió los costos de la preparación de colon o de traslado.

accedió inicialmente, pero no asistió, también se le interrogó por qué no acudió, obteniendo respuestas similares a las del grupo que declinó al inicio (Tabla 1).

Del total de individuos contactados, 76 (31\%) se estudiaron con colonoscopia (considerando a los individuos asintomáticos que ya se habían controlado). En la Tabla 2 se resumen las características del grupo sometido a estudio.

Se logró una colonoscopia hasta el ciego en 75 de los 76 pacientes y no hubo complicaciones en ninguno de ellos. En 16 pacientes, se observó pólipos en el colon, 13 adenomatosos (17\%) (doce pacientes presentaron adenomas tubulares y a uno se le diagnosticó cáncer). En tres pacientes se hizo el diagnóstico de pólipos hiperplásticos y en los restantes 60 , no se evidenció tumores (Tabla 3). Los pacientes con neoplasias tuvieron una edad media
Tabla 2 C aracterísticas del grupo sometido a estudio

\begin{tabular}{|lrr|}
\hline & $\mathrm{n}$ & $\%$ \\
\hline Sexo masculino & 47 & 61,8 \\
Grupo etáreo & & \\
$\quad$ Menor 30 & 1 & 1,3 \\
$31-40$ & 14 & 18,4 \\
$41-50$ & 30 & 39,5 \\
$51-60$ & 18 & 23,7 \\
$61-70$ & 13 & 17,1 \\
Total & 76 & 100 \\
\hline
\end{tabular}

de 54,8 años con un rango entre 42 y 73 años, cuatro tenían una edad menor a 50 años (diagnóstico de adenomas a los 42, 43, 44 y 46 años).

Tabla 3. Resultados de la colonoscopia en familiares de primer grado de pacientes operados por cáncer colorrectal

\begin{tabular}{|lrr|}
\hline & $\mathrm{n}$ & $\%$ \\
\hline Individuos estudiados & 76 & 100 \\
Colonoscopia hasta el ciego & 75 & 99 \\
Complicaciones & 0 & 0 \\
Individuos con pólipos: & 16 & 21,1 \\
$\quad$ Únicos & 13 & 17,1 \\
$\quad 3$ & 3,9 \\
Múltiples & 3 & 17,1 \\
$\quad 13$ & 15,8 \\
$\quad$ con pólipos adenomatosos & 12 & 1,3 \\
con adenocarcinoma & 1 & 5,3 \\
\hline Individuos con pólipos hiperplásticos & 4 & \\
\hline
\end{tabular}


La distribución de los pólipos fue homogénea en todo el intestino, cinco de ellos se ubicaban en el colon derecho y tres no se asociaban a tumores en el colon distal.

\section{DISCUSIÓN}

En este estudio se diagnosticaron neoplasias en $17 \%$ de los individuos estudiados, resultado que es comparable con otras experiencias similares cuyas tasas de detección varían entre $10 \%$ y $25 \%$, Tabla $4^{10-14}$. Considerando el alza en la mortalidad por esta patología en nuestro país, creemos que el hallazgo de neoplasias observadas en este estudio, permite recomendar a los médicos de atención primaria la indicación de estudio a la población con antecedentes de familiares de primer grado con cáncer colorrectal.

El hecho de que tres adenomas tubulares se hayan diagnosticado en el colon derecho sin tumores sincrónicos en colon izquierdo, plantea la importancia de una exploración completa del colon para permitir el diagnóstico y tratamiento de las lesiones proximales, la que no es posible con exámenes más abreviados como la sigmoidoscopia o la colonoscopia izquierda que serían, por lo tanto, exámenes inapropiados para esta población. Hunt LM et $\mathrm{al}^{11}$ en una experiencia similar a la aquí presentada mostró que $26 \%$ de los tumores diagnosticados se encontraban en el ángulo esplénico o proximal a éste.

Destacamos el hecho de que casi un tercio de los pacientes con diagnóstico de neoplasias en la colonoscopia fueran menores de 50 años. Esto es concordante con lo que muestran otros estudios extranjeros, que concluyen que la historia familiar de cáncer colorrectal se asocia a un riesgo aumentado de la enfermedad, especialmente en personas menores de 50 años ${ }^{15}$. La recomendación de colonoscopia en los familiares desde los 40 años, ha sido respaldada por el hallazgo de que la curva de incidencia por edad de cáncer de colon en esta población se adelanta en 10 años a la población general. Se sabe también que el riesgo aumentado en esta población ocurre principalmente antes de los 45 años, donde el riesgo relativo llega hasta 5,4. Después de esta edad el riesgo se va aproximando gradualmente al de la población general ${ }^{15}$. Es importante destacar que la mayoría de las neoplasias malignas del colon se originan en pólipos cuya transición al cáncer puede tomar entre 6-10 años. Es en este periodo en el cual debieran efectuarse las medidas de detección precoz y tratamiento de las lesiones premalignas para así abortar la secuencia adenoma-carcinoma.

En nuestro equipo, desde comienzos de los años 90, rutinariamente se entregan trípticos educativos a los pacientes con neoplasias del colon, que incluyen información sobre el riesgo familiar de esta enfermedad. Sin embargo, inquietos por el bajo porcentaje de familiares que asisten al estudio, se decidió realizar este protocolo, en el cual un grupo de enfermeras independientemente del médico tratante, contactaron a los familiares en dos oportunidades. A pesar del esfuerzo desplegado (tríptico educativo, contacto telefónico y examen gratuito) la adherencia fue baja, pues más de dos tercios de los familiares no acudieron a realizarse el examen. Esto nos lleva a pensar en

\section{Tabla 4. Experiencias de estudio con colonoscopia en familiares asintomáticos de primer grado de pacientes con cáncer colorrectal}

\begin{tabular}{|lccc|}
\hline Referencia & $\mathrm{n}$ & $\begin{array}{c}\text { Hallazgo de neoplasia } \\
\text { adenomas o cáncer }\end{array}$ & Detección de cáncer \\
\hline Hunt LM et al 1998 & 330 & $40(12 \%)$ & 0 \\
Gilbert JM et al 2001 & 212 & $54(25,5 \%)$ & 0 \\
Wu CS et al 1995 & 213 & $26(12,2 \%)$ & $5(2,3 \%)$ \\
Clark SK et al 2003 & 186 & $24(12,9 \%)$ & $3(1,6 \%)$ \\
Syrigos KN et al 2002 & 249 & $27(10,8 \%)$ & 0 \\
López et al 2006 & 76 & $13(17,1 \%)$ & $1(1,3 \%)$ \\
\hline
\end{tabular}


dos direcciones: temor a la incomodidad del examen utilizado y/o falta de conciencia de salud en la población. Se debe destacar que a los pacientes que formalmente se negaron a efectuarse la colonoscopia, se les recomendó el estudio de sangre oculta en las deposiciones, examen que tampoco se practicaron (datos no mostrados).

Otro hecho interesante es que casi dos tercios del grupo estudiado estuvo constituido por individuos que ya se habían examinado por iniciativa propia, previo al contacto telefónico y a pesar de no tener síntomas, probablemente motivados por

\section{REFERENCIAS}

1. Vines JJ, Ardanaz E, Arrazola A, Gaminde I. Population-based epidemiology of colorectal cancer: causality review. An Sist Sanit Navar 2003; 26: 79-97.

2. Donoso A, Viluarroel L, Pinedo G. Aumento de la mortalidad por cáncer de colon en Chile, 19902003. Rev Méd Chile 2006; 134: 152-8.

3. Parker S, Tong T, Bolden S, Wingo P. Cancer statistics, 1996. CA Cancer J Clin 1996; 46: 5-27.

4. Winawer S, Fietcher R, Rex D, Bond J, Burt R, FERRUCCI J ET AL. Colorectal cancer screening and surveillance: clinical guidelines and rationaleUpdate based on new evidence. Gastroenterology 2003; 124: 544-60.

5. Pignone M, Saha S, Hoerger T, Mandeiblatt J. Costeffectiveness analyses of colorectal cancer screening: a systematic review for the U.S. Preventive Services Task Force. Ann Intern Med 2003; 137: 96-104.

6. Walsh JM, TeRdiman JP. Colorectal cancer screening: clinical applications. Jama 2003; 289: 1297302.

7. Jemal A, Thomas A, Murray T, Thun M. Cancer statistics, 2002. CA Cancer J Clin 2002; 52: 23-47.

8. Vogelstein B, Fearon ER, Hamilon SR, Kern SE, PREISINGER AC, LEPPERT M ET AL. Genetic alterations during colorectal-tumor development. N Engl J Med 1988; 319: 525-32. la información entregada por cada médico tratante a su familiar con cáncer. Según esto, cabe suponer que una estrategia de educación sencilla como la entrega de material escrito a los pacientes con cáncer puede ser eficaz en lograr adherencia en sus familiares a métodos de estudio.

Finalmente, debemos destacar la responsabilidad de todos los médicos y autoridades en apoyar y fomentar iniciativas de prevención oncológicas las cuales contribuirán en generar mayor conciencia en la población y controlar una grave patología que va en aumento.

9. Winawer S, Zauber A, Ho M, O'Brien M, Gottlieb L, SteRnBERg $S$ et al. Prevention of colorectal cancer by colonoscopic polypectomy. The National Polyp Study Workgroup. N Engl J Med 1993; 329: 1977-81.

10. Wu CS, Tung SY, Chen PC, Kuo YC. The role of colonoscopy in screening persons with family history of colorectal cancer. J Gastroenterol Hepatol 1995; 10: 319-23.

11. Hunt LM, Rooney PS, Hardcastle JD, Armitage NC. Endoscopic screening of relatives of patients with colorectal cancer. Gut 1998; 42: 71-5.

12. Gilbert JM, Vaizey CJ, Cassell PG, Holden J. Feasibility study of colonoscopy as the primary screening investigation in relatives of patients with colorectal cancer. Ann R Coll Surg Engl 2001; 83: 415-9.

13. Ciark SK, Carpenter S, Broughton CI, Marks CG. Surveillance of individuals at intermediate risk of colorectal cancer. The impact of new guidelines. Colorectal Dis 2003; 5: 582-4.

14. Syrigos KN, Charalampopoulos A, Ho JL, Zbar A, MURDAY VA, LEICESTER RJ. Colonoscopy in asymptomatic individuals with a family history of colorectal cancer. Ann Surg Oncol 2002; 9: 439-43.

15. Fuchs C, Giovannucci E, Colditz G, Hunter D, SPEIZER F, WILETT W. A prospective study of family history and the risk of colorectal cancer. N Engl J Med 1994; 331: 1669-74. 